Kragujevac Journal of Mathematics

Volume 45(3) (2021), Pages 343-352.

\title{
SERIES EXPANSION OF A COTANGENT SUM RELATED TO THE ESTERMANN ZETA FUNCTION
}

\author{
MOULOUD GOUBI ${ }^{1}$
}

\begin{abstract}
In this paper, we study the cotangent sum $c_{0}\left(\frac{q}{p}\right)$ related to the Estermann zeta function for the special case when the numerator is equal to 1 and get two useful series expansions of $c_{0}\left(\frac{1}{p}\right)$.
\end{abstract}

\section{INTRODUCTION}

For a positive integer $p$ and $q=1,2, \ldots, p-1$, such that $(p, q)=1$, let the cotangent sum (see [10])

$$
c_{0}\left(\frac{q}{p}\right)=-\sum_{k=1}^{p-1} \frac{k}{p} \cot \frac{\pi k q}{p} .
$$

$c_{0}\left(\frac{q}{p}\right)$ is the value at $s=0$,

$$
E_{0}\left(0, \frac{q}{p}\right)=\frac{1}{4}+\frac{i}{2} c_{0}\left(\frac{q}{p}\right)
$$

of the Estermann zeta function

$$
E_{0}\left(s, \frac{q}{p}\right)=\sum_{k \geq 1} \frac{d(k)}{k^{s}} \exp \left(\frac{2 \pi i k q}{p}\right) .
$$

It is well-known that the sum $c_{0}\left(\frac{q}{p}\right)$ satisfies the reciprocity formula (see [2])

$$
c_{0}\left(\frac{q}{p}\right)+\frac{p}{q} c_{0}\left(\frac{p}{q}\right)-\frac{1}{\pi q}=\frac{i}{2} \psi_{0}\left(\frac{q}{p}\right) .
$$

Key words and phrases. Estermann zeta function, Vasyunin cotangent sum, generating function. 2010 Mathematics Subject Classification. Primary: 11F20, 11E45. Secondary: 11M26.

DOI 10.46793/KgJMat2103.343G

Received: October 06, 2018.

Accepted: January 08, 2019. 
The Vasyunin cotangent sum (see [11])

$$
V\left(\frac{q}{p}\right)=\sum_{r=1}^{p-1}\left\{\frac{r q}{p}\right\} \cot \left(\frac{\pi r}{p}\right)=-c_{0}\left(\frac{\bar{q}}{p}\right)
$$

arises in the study of the Riemann zeta function by virtue of the formula (see $[2,9]$ )

$$
\begin{aligned}
& \frac{1}{2 \pi \sqrt{p q}} \int_{-\infty}^{+\infty}\left|\zeta\left(\frac{1}{2}+i t\right)\right|^{2}\left(\frac{q}{p}\right)^{i t} \frac{d t}{\frac{1}{4}+t^{2}} \\
= & \frac{\log 2 \pi-\gamma}{2}\left(\frac{1}{p}+\frac{1}{q}\right)+\frac{p-q}{2 p q} \log \frac{q}{p}-\frac{\pi}{2 p q}\left(V\left(\frac{p}{q}\right)+V\left(\frac{q}{p}\right)\right) .
\end{aligned}
$$

This formula is connected to the approach of Nyman, Beurling and Báez-Duarte to the Riemann hypothesis (see [8]), which states that the Riemann hypothesis is true if and only if $\lim _{n \rightarrow \infty} d_{N}=0$, where

$$
d_{N}^{2}=\inf _{A_{N}} \frac{1}{2 \pi} \int_{-\infty}^{+\infty}\left|1-\zeta A\left(\frac{1}{2}+i t\right)\right|^{2} \frac{d t}{\frac{1}{4}+t^{2}},
$$

and the infimum is taken over all Dirichlet polynomials

$$
A_{N}(s)=\sum_{n=1}^{N} \frac{a_{n}}{n^{s}}
$$

In a recent work with A. Bayad [7], we have proved that the sum $V\left(\frac{q}{p}\right)$ satisfies the reciprocity formula

$$
V\left(\frac{q}{p}\right)+V\left(\frac{p}{q}\right)=\frac{1}{\pi}\left(G(p, p)+G(q, q)+G(p, q)+(q-p) \log \frac{q}{p}\right),
$$

where

$$
G(p, q)=\sum_{k \geq 1} \frac{p q}{k(k+1)}\left\{\frac{k}{p}\right\}\left\{\frac{k}{q}\right\} .
$$

Thereafter the restriction of the relationship (1.1) to $q=1$ gives

$$
c_{0}\left(\frac{1}{p}\right)=-\frac{1}{\pi} G(p, p)-(p-1) \log p .
$$

Exactly our interest in this work is the case $q=1$ in order to get two series expansions of $c_{0}\left(\frac{1}{p}\right)$. First we recall the different asymptotical writings of $c_{0}\left(\frac{1}{p}\right)$ in the literature. In [10, Theorem 1.2, Theorrem 1.3] M. Th. Rassias proved that

$$
c_{0}\left(\frac{1}{p}\right)=\frac{1}{\pi} p \log p-\frac{p}{\pi}(\log 2 \pi-\gamma)+\{\mathcal{O}(\log p) \text { or } \mathcal{O}(1)\} .
$$

In [9, Theorem 1.7] H. Maier and M. Th. Rassias provide the following improvement. Let $b, n \in \mathbb{N}, b \geq 6 N$, with $N=\left\lfloor\frac{n}{2}\right\rfloor+1$. There exist absolute real constants $A_{1}, A_{2} \geq 1$ 
and absolute real constants $E_{l}, l$, with $\left|E_{l}\right| \leq\left(A_{1} l\right)^{2 l}$, such that for each $n \in \mathbb{N}$ we have

$$
c_{0}\left(\frac{1}{p}\right)=\frac{1}{\pi} p \log p-\frac{p}{\pi}(\log 2 \pi-\gamma)-\frac{1}{\pi}+\sum_{l=1}^{n} E_{l} p^{-l}+R_{n}^{\star}(p),
$$

where $\left|R_{n}^{\star}(p)\right| \leq\left(A_{2} n\right)^{4 n} p^{-(n+1)}$.

Only in [9, Theorem 1.9] H. Maier and M. Th. Rassias provide another improvement,

$$
c_{0}\left(\frac{1}{p}\right)=\frac{1}{\pi} p \log p-\frac{p}{\pi}(\log 2 \pi-\gamma)+C_{1} p+\mathcal{O}(1) .
$$

We draw attention that $\mathrm{S}$. Bettin finds other reformulations of $c_{0}\left(\frac{1}{p}\right)$ inspired from continued fraction theory (see [3]).

Finally from another point of view we show in [5] with A. Bayad and M. O. Hernane that

$$
\begin{aligned}
c_{0}\left(\frac{1}{p}\right)= & -\frac{1}{\pi}\left(\log \frac{2 \pi}{p}-\gamma\right) p+\frac{1}{\pi}+\frac{\pi}{36 p} \\
& -\frac{1}{2} \sum_{k=2}^{\left\lfloor\frac{N}{2}\right\rfloor}(-1)^{k} \frac{4^{k} \pi^{2 k-1} B_{2 k}^{2}}{k(2 k) !}\left(\frac{1}{p}\right)^{2 k-1}+\mathcal{O}\left(\frac{1}{p^{N}}\right) .
\end{aligned}
$$

There is a misprint in the formula (1.22) Corollary 1.2 in [5] the correct one is in the formula (1.21) Corollary 1.2.

Otherwise in the same paper [5], an integral representation of $c_{0}\left(\frac{1}{p}\right)$ is given by

$$
c_{0}\left(\frac{1}{p}\right)=\frac{1}{\pi} \int_{0}^{1} \frac{(p-2) x^{p}-p x^{p-1}+p x-p+2}{(x-1)^{2}\left(x^{p}-1\right)} d x .
$$

In this work we prove that

$$
(p-2) x^{p}-p x^{p-1}+p x-p+2=(x-1)^{3} \sum_{r=1}^{p-1}(p-r-1) r x^{r-1}
$$

and we get another formulation that is

$$
c_{0}\left(\frac{1}{p}\right)=\frac{1}{\pi} \int_{0}^{1} \frac{\sum_{r=1}^{p-1}(p-r-1) r x^{r-1}}{1+x+\cdots+x^{p-1}} d x .
$$

Applying some techniques from the generating function theory [4] to previous integrals; we find two series expansions of $c_{0}\left(\frac{1}{p}\right)$, as they are well explained in the next section.

\section{SERIES EXPANSION OF $c_{0}\left(\frac{1}{p}\right)$}

Let $b_{k}$ be the integer sequence defined by $b_{0}=1, b_{1}=2$ and the recursive formulae:

$$
\begin{gathered}
b_{k}-2 b_{k-1}+b_{k-2}=0, \quad 2 \leq k \leq p-1, k=p+1, \\
b_{p}-2 b_{p-1}+b_{p-2}=1
\end{gathered}
$$


and

$$
b_{k}-2 b_{k-1}+b_{k-2}-b_{k-p}+2 b_{k-p-1}-b_{k-p-2}=0, \quad k \geq p+2 .
$$

According to the terms $b_{k}$ we get the first series expansion in the following theorem.

\section{Theorem 2.1.}

$$
c_{0}\left(\frac{1}{p}\right)=\frac{1}{\pi} p(p-1)(p-2) \sum_{k \geq 0} \frac{b_{k}}{(k+1)(k+p+1)(k+2)(k+p)} .
$$

For $p \geq 1$ we define the arithmetic function $a_{p}$ in the form

$$
a_{p}(k)= \begin{cases}1, & \text { if } p \mid k \\ -1, & \text { if } k \equiv 1 \quad(\bmod p) \\ 0, & \text { otherwise }\end{cases}
$$

This function is not multiplicative. In general the arithmetical functions are defined from the set of natural integers $\mathbb{N}$ into $\mathbb{C}$. We can extend this definition to $\mathcal{F}(\mathbb{C}, \mathbb{C})$; set of functions from $\mathbb{C}$ to $\mathbb{C}$. In that case the corresponding function is $A: \mathbb{N} \rightarrow \mathcal{F}(\mathbb{C}, \mathbb{C})$ with $A(p)=a_{p}$. Furthermore, $A(p q)= \pm A(p) A(q)$ and $|A|$ is multiplicative.

Let the function $M(p, k)$ defined by

$$
M(p, 0)=\frac{1}{2} p^{2}-\frac{3}{2} p+1
$$

and

$$
M(p, k)=(p-1)\left(\frac{1}{2} p+k-1\right)-k(p+k-1)\left(H_{p+k-1}-H_{k}\right), \quad k \geq 1,
$$

where $H_{k}$ is the Harmonic number

$$
H_{k}=\sum_{j=1}^{k} \frac{1}{j}
$$

Following this function a second series expansion of $c_{0}\left(\frac{1}{p}\right)$ is given in the following theorem.

\section{Theorem 2.2.}

$$
c_{0}\left(\frac{1}{p}\right)=\frac{1}{\pi} \sum_{k \geq 0} a_{p}(k) M(p, k) .
$$

2.1. Proof of Theorem 2.1. We take inspiration from the theory of generating functions $[4,6]$, and prove that the sequence $\left(b_{k}\right)$ is generated by the rational function:

$$
f(x)=\frac{1}{1-2 x+x^{2}-x^{p}+2 x^{p+1}-x^{p+2}} .
$$

More precisely we get the following lemma.

\section{Lemma 2.1.}

$$
\frac{1}{1-2 x+x^{2}-x^{p}+2 x^{p+1}-x^{p+2}}=\sum_{k \geq 0} b_{k} x^{k}, \quad|x|<1 .
$$


Proof. It is well known that

$$
\frac{1}{1-x}=\sum_{k \geq 0} x^{k}, \quad|x|<1
$$

Since for $0 \leq x<1$

$$
0<(x-1)^{2}\left(1-x^{p}\right)<1
$$

and

$$
(x-1)^{2}\left(1-x^{p}\right)=1-\left(2 x-x^{2}+x^{p}-2 x^{p+1}+x^{p+2}\right),
$$

then we have

$$
0<2 x-x^{2}+x^{p}-2 x^{p+1}+x^{p+2}<1 .
$$

Furthermore, $f(x)$ is developable on entire series to get the result we have to take the quantity $2 x-x^{2}+x^{p}-2 x^{p+1}+x^{p+2}$ instead of $x$ in the last formula (2.4). Now, writing

and then

$$
\frac{1}{1-2 x+x^{2}-x^{p}+2 x^{p+1}-x^{p+2}}=\sum_{k \geq 0} d_{k} x^{k}
$$

$$
\left(1-2 x+x^{2}-x^{p}+2 x^{p+1}-x^{p+2}\right)\left(\sum_{k \geq 0} d_{k} x^{k}\right)=1 .
$$

To compute this we use the well known Cauchy product of two entire series

$$
\left(\sum_{k \geq 0} a_{k} x^{k}\right)\left(\sum_{j \geq 0} d_{j} x^{j}\right)=\sum_{k \geq 0}\left(\sum_{j=0}^{k} a_{j} d_{k-j}\right) x^{k},
$$

which generates the product of a polynomial of degree $n$ with an entire series that also gives an entire series as follows

$$
\left(\sum_{k=0}^{n} a_{k} x^{k}\right)\left(\sum_{j \geq 0} d_{j} x^{j}\right)=\sum_{k \geq 0}\left(\sum_{j=0}^{\min \{n, k\}} a_{j} d_{k-j}\right) x^{k} .
$$

We return to $f(x)$ in writing

$$
1-2 x+x^{2}-x^{p}+2 x^{p+1}-x^{p+2}=\sum_{k=0}^{p+2} a_{k} x^{k},
$$

with $a_{0}=1, a_{1}=-2, a_{2}=1, a_{p}=-1, a_{p+1}=2, a_{p+2}=-1$, and the others are zero. We conclude that $d_{0}=1, d_{1}=2$. The formula

$$
\sum_{j=0}^{\min \{p+2, k\}} a_{j} d_{k-j}=0
$$

states that

$$
\begin{gathered}
d_{k}-2 d_{k-1}+d_{k-2}=0, \quad 2 \leq k \leq p-1, \quad k=p+1, \\
d_{p}-2 d_{p-1}+d_{p-2}=1
\end{gathered}
$$

and

$$
d_{k}-2 d_{k-1}+d_{k-2}-d_{k-p}+2 d_{k-p-1}-d_{k-p-2}=0, \quad k \geq p+2 .
$$


Finally, we see that $d_{k}$ and $b_{k}$ are identical for every integer $k \geq 0$. For more information on this approach we refer to [6].

To get the result (2.1) of Theorem 2.1 we must substitute the expression (2.3) in the identity (1.2) and one obtains

$$
c_{0}\left(\frac{1}{p}\right)=-\frac{1}{\pi} \sum_{k \geq 0} b_{k} \int_{0}^{1}\left((p-2) x^{k+p}-p x^{k+p-1}+p x^{k+1}+(2-p) x^{k}\right) d x .
$$

Furthermore,

$$
c_{0}\left(\frac{1}{p}\right)=-\frac{1}{\pi} \sum_{k \geq 0} b_{k}\left(\frac{p-2}{k+p+1}-\frac{p}{k+p}+\frac{p}{k+2}-\frac{p-2}{k+1}\right) .
$$

Finally,

$$
c_{0}\left(\frac{1}{p}\right)=\frac{1}{\pi} p(p-1)(p-2) \sum_{k \geq 0} \frac{b_{k}}{(k+1)(k+p+1)(k+2)(k+p)}
$$

and $c_{0}(1)=c_{0}\left(\frac{1}{2}\right)=0$ is compatible with the definition of $c_{0}$.

Regarding the identity (2.3) Lemma 2.1 we remark that

$$
\frac{1}{(1-x)^{2}\left(1-x^{p}\right)}=\sum_{k \geq 0} b_{k} x^{k}, \quad|x|<1 .
$$

Furthermore, for $x=\frac{1}{2}$ we deduce that the coefficients $b_{k}$ satisfy the following statements

$$
\sum_{k \geq 0} \frac{b_{k}}{2^{k}}=\frac{2^{p+2}}{2^{p}-1} \quad \text { and } \quad \lim _{k \rightarrow \infty} \frac{b_{k}}{2^{k}}=0 .
$$

2.2. Proof of Theorem 2.2. First we began by proving another integral representation of $c_{0}\left(\frac{1}{p}\right)$.

\section{Lemma 2.2.}

$$
c_{0}\left(\frac{1}{p}\right)=\frac{1}{\pi} \int_{0}^{1} \frac{\sum_{r=1}^{p-1}(p-r-1) r x^{r-1}}{1+x+\cdots+x^{p-1}} d x .
$$

Proof.

$$
\begin{aligned}
(x-1)^{3} \sum_{r=1}^{q-1}(q-r-1) r x^{r-1}= & \sum_{r=3}^{q}(q-r+1)(r-2) x^{r}-3 \sum_{r=2}^{q-1}(q-r)(r-1) x^{r} \\
& +3 \sum_{r=1}^{q-2}(q-r-1) r x^{r}-\sum_{r=0}^{q-3}(q-r-2)(r+1) x^{r} .
\end{aligned}
$$

It's obvious to remark that

$$
(q-r+1)(r-2)-3(q-r)(r-1)+3(q-r-1) r-(q-r-2)(r+1)=0
$$


and the quantity

$$
(t-1)^{3} \sum_{r=1}^{q-1}(q-r-1) r x^{r-1}
$$

is reduced to

$$
\begin{aligned}
& (q-2) x^{q}+2(q-3) x^{q-1}+3(q-4) x^{q-2}-3(q-2) x^{q-1}-6(q-3) x^{q-2}-3(q-2) x^{2} \\
& +3(q-2) x^{q-2}+3(q-2) x+6(q-3) x^{2}-q+2-2(q-3) x-3(q-4) x^{2} .
\end{aligned}
$$

After simplification we obtain

$$
(t-1)^{3} \sum_{r=1}^{q-1}(q-r-1) r x^{r-1}=(q-2) x^{q}-q x^{q-1}+q x-q+2 .
$$

The Theorem 2.2 is immediate from the Lemma 2.2 in the following way. Since

$$
\frac{1}{1+x+\cdots+x^{p-1}}=\frac{1-x}{1-x^{p}}
$$

and $|x|<1$, then

$$
\frac{1}{1+x+\cdots+x^{p-1}}=\frac{1-x}{1-x^{p}}=\sum_{k \geq 0}(1-x) x^{p k} .
$$

Furthermore,

$$
\frac{1}{1+x+\cdots+x^{p-1}}=\sum_{k \geq 0} a_{p}(k) x^{k}
$$

and we have

$$
\frac{\sum_{r=1}^{p-1}(p-r-1) r x^{r-1}}{1+x+\cdots+x^{p-1}}=\sum_{k \geq 0} \sum_{r=1}^{p-1} a_{p}(k)(p-r-1) r x^{k+r-1} .
$$

The passage to the integral inducts

$$
c_{0}\left(\frac{1}{p}\right)=\sum_{k \geq 0} \sum_{r=1}^{p-1} a_{p}(k) \frac{(p-r-1) r}{k+r} .
$$

But

$$
\sum_{r=1}^{p-1} \frac{(p-r-1) r}{k+r}=(p-1)\left(\frac{1}{2} p+k-1\right)-k(p+k-1) \sum_{r=k+1}^{p+k-1} \frac{1}{r}
$$

and the result (2.2) is deduced. 


\section{Connection to Digamma Function}

We finish this work by revisiting the proof of the expression of $c_{0}\left(\frac{1}{p}\right)$ according to the function digamma and Bernoulli polynomials in the work [1] of L. Báez Duarte et al.

$$
c_{0}\left(\frac{1}{p}\right)=\frac{2}{\pi} \sum_{r=1}^{p-1} B_{1}\left(\frac{r}{p}\right) \psi\left(\frac{r}{p}\right),
$$

where $B_{1}$ is the reduced Bernoulli polynomial

$$
B_{1}(x)= \begin{cases}0, & \text { if } x \in \mathbb{Z} \\ \{x\}-\frac{1}{2}, & \text { otherwise }\end{cases}
$$

and $\psi$ the digamma function defined by

$$
\psi(z)=-\gamma-\frac{1}{z}+\sum_{k \geq 1}\left(\frac{1}{k}-\frac{1}{k+z}\right) .
$$

Starting with the demonstration of a property of $\psi$ that will be used later.

\section{Proposition 3.1.}

$$
\psi\left(\frac{r+1}{p}\right)-\psi\left(\frac{r}{p}\right)=p \int_{0}^{1} \frac{x^{r-1}}{1+x+\cdots+x^{p-1}} d x .
$$

Proof. We quote from [5] the formula

$$
\psi\left(\frac{r+1}{p}\right)-\psi\left(\frac{r}{p}\right)=p \sum_{k \geq 0} \frac{1}{(p k+r+1)(p k+r)} .
$$

The general term $\frac{1}{(p k+r+1)(p k+r)}$ can be written as following

$$
\frac{1}{(p k+r+1)(p k+r)}=\frac{1}{p k+r}-\frac{1}{p k+r+1}=\int_{0}^{1}\left(x^{p k+r-1}-x^{p k+r}\right) d x
$$

and the passage to the sum states that

$$
\sum_{k \geq 0} \frac{1}{(p k+r+1)(p k+r)}=\int_{0}^{1} \frac{x^{r-1}-x^{r}}{1-x^{p}} d x .
$$

Finally,

$$
\sum_{k \geq 0} \frac{1}{(p k+r+1)(p k+r)}=\int_{0}^{1} \frac{x^{r-1}}{1+x+\cdots+x^{p-1}} d x
$$

and we have (3.1). Proposition 3.1 follows.

In [5], it is shown that

$$
\log p=\frac{1}{p} \sum_{r=1}^{p-1} r\left(\psi\left(\frac{r+1}{p}\right)-\psi\left(\frac{r}{p}\right)\right) .
$$

This identity conducts to the following interesting lemma. 


\section{Lemma 3.1.}

$$
\sum_{r=1}^{p} \psi\left(\frac{r}{p}\right)=-\gamma p-p \log p
$$

Proof. Since

$$
\sum_{r=1}^{p-1} r\left(\psi\left(\frac{r+1}{p}\right)-\psi\left(\frac{r}{p}\right)\right)=p \log p
$$

then

$$
-\sum_{r=1}^{p} \psi\left(\frac{r}{p}\right)+\psi(1) p=p \log p
$$

Furthermore,

$$
\sum_{r=1}^{p} \psi\left(\frac{r}{p}\right)=-\gamma p-p \log p
$$

According to the identity (3.1) Proposition 3.1 and the integral representation (2.5) we conclude that

$$
c_{0}\left(\frac{1}{p}\right)=\frac{1}{\pi p} \sum_{r=1}^{p-1}(p-r-1) r\left(\psi\left(\frac{r+1}{p}\right)-\psi\left(\frac{r}{p}\right)\right) .
$$

Furthermore combining this result with the identity (3.2) Lemma 3.1 we get

$$
c_{0}\left(\frac{1}{p}\right)=-\frac{1}{\pi} \log p+\frac{1}{\pi p} \sum_{r=1}^{p-1}(p-r) r\left(\psi\left(\frac{r+1}{p}\right)-\psi\left(\frac{r}{p}\right)\right)
$$

and

$$
c_{0}\left(\frac{1}{p}\right)=-\frac{1}{\pi} \log p-\gamma \frac{p-1}{\pi p}+\frac{1}{\pi p} \sum_{r=1}^{p-1}(2 r-p-1) \psi\left(\frac{r}{p}\right)
$$

then

$$
c_{0}\left(\frac{1}{p}\right)=\frac{1}{\pi p} \sum_{r=1}^{p-1}(2 r-p) \psi\left(\frac{r}{p}\right) .
$$

But

$$
2 r-p=2 p\left(\frac{r}{p}-\frac{1}{2}\right)=2 p B_{1}\left(\frac{r}{p}\right)
$$

which means that

$$
c_{0}\left(\frac{1}{p}\right)=\frac{2}{\pi} \sum_{r=1}^{p} B_{1}\left(\frac{r}{p}\right) \psi\left(\frac{r}{p}\right) .
$$




\section{REFERENCES}

[1] L. Báez Duarte, M. Balazard, M. Landreau and E. Saias, Etude de l'autocorrélation multiplicative de la fonction partie fractionnaire, Ramanujan J. 9 (2005), 215-240.

[2] S. Bettin and J. B. Conrey, Period functions and cotangent sums, Algebra and Number Theory 7(1) (2013), 215-242.

[3] S. Bettin, On the distribution of a cotangent sum, Int. Math. Res. Not. IMRN 2015(21) (2015), 11419-11432.

[4] G. B. Djordjević and G. V. Milovanović, Special Classes of Polynomials, University of Niš, Faculty of Technology, Leskovac, 2014.

[5] M. Goubi, A. Bayad and M. O. Hernane, Explicit and asymptotic formulae for Vasyunin-cotangent sums, Publ. Inst. Math. (Beograd) (N.S.) 102(116) (2017), 155-174.

[6] M. Goubi, Successive derivatives of Fibonacci type polynomials of higher order in two variables, Filomat 32(4) (2018), 5149-5159.

[7] A. Bayad and M. Goubi, Reciprocity formulae for generalized Dedekind-Vasyunin-cotangent sums, Math. Methods Appl. Sci. 42(4) (2019), 1082-1098.

[8] H. Maier, M. Th. Rassias, Explicit estimates of sums related to the Nyman-Beurling criterion for the Riemann hypothesis, J. Funct. Anal. DOI 10.1016/j.jfa.2018.06.022.

[9] H. Maier, M. Th. Rassias, Generalizations of a cotangent sum associated to the Estermann zeta function, Commun. Contemp. Math. 18(1) (2016), DOI 10.1142/S0219199715500789.

[10] M. Th. Rassias, A cotangent sum related to zeros of the Estermann zeta function, Appl. Math. Comput. 240 (2014), 161-167.

[11] V. I. Vasyunin, On a biorthogonal system associated with the Riemann hypothesis, Algebra i Analiz 7(3) (1995), 118-135.

${ }^{1}$ Department of Mathematics, UNIVERSiTY OF MOUlOUd MAMMERI, Hasnaoua II Tizi-Ouzou 15000 Algeria

Email address: mouloud.ummto@hotmail.fr 